\title{
Sustaining Bioethical Contributions in Times of Crisis and Change
}

\section{Graeme Laurie $^{1}$}

Published online: 10 June 2020

(C) National University of Singapore and Springer Nature Singapore Pte Ltd. 2020

In this first issue of the Asian Bioethics Review since the advent of global lockdown as a result of the COVID-19 pandemic, we are very pleased to publish a range of papers from across the globe that demonstrate very admirably the continued contributions of the bioethics community. We have been particularly heartened by the very strong response to our Call for Perspective papers addressing the ethical issues raised by the pandemic. In addition to the excellent tranche of articles outlined below, we are confident of being able to secure significant space in all future issues of 2020 - and beyond if necessary - to publish colleagues' insights on how we are responding to this global public health emergency.

The current issue is framed by COVID-19-related contributions, but we also offer other important perspectives in bioethics on a range of issues - all advocating for change in our world. As to the pandemic, we open with an original article by Xafis et al. (2020) that provides a comprehensive account of the diverse ethical issues that arise, and importantly it scopes how these extend into many areas of life and global concern, including to climate change. Later in the issue, we offer the first set of Perspectives papers that focus intently on key questions or aspects of the pandemic. Gopichandran et al. (2020) provide a foundational analysis of the ways in which a pandemic such as COVID-19 pushes public trust in health systems to its limits; they offer valuable lessons from the experiences of Tamil Nadu, India, in this regard - some very positive and others less so. From key members of the medical community in Singapore, we are pleased to publish the Joint Position Statement of the Singapore Hospice Council and the Chapter of Palliative Medicine Physicians on the role of palliative medicine in ICU bed allocation during the pandemic (Krishna et al. 2020). This is a fascinating example of ethics-in-action, both for its illustration of how key ethical principles inform decision-making and as an example of a profession's ethic of care extending beyond the immediate health needs of patients: the Statement talks of the importance of providing family support, as well as the need to consider extended care responsibilities when a decision against ICU is taken. Indeed, reminding us of the core ethical issues at stake in this kind of decision-making, Xafis (2020) then provides

Graeme Laurie

Graeme.Laurie@ed.ac.uk

1 Edinburgh Law School, The University of Edinburgh, Edinburgh, UK 
an account of likely health inequalities relating to public health emergencies, bringing well-established ethical analysis to bear on the current crisis. The risk of stigmatisation and discrimination is the focus of the paper by Cave (2020), who deconstructs the concept of the 'super spreader'. While noting that this expression has valid and enduring scientific meaning, she highlights that its adoption in wider social settings can lead - and has led - to unethical treatment of individuals to whom the label is attached. She calls for a careful re-examination of the term, and cautions against its unreflexive use. Finally, in a further call for more reflexive practice, Lim (2020) offers an account of the implications of applying MacIntyre's work on the common good to lessons from the pandemic, notably the prevalent reaction of many individuals to ignore social distancing policies or other measures designed to promote public health. Lim argues that this phenomenon can be linked, in part, to the hold that neo-liberal philosophies now have in many countries of the globe. As a response, she advocates integrating McIntyre's philosophy on the values underpinning the common good into education and medical ethics training.

Beyond these COVID-19 contributions, the journal remains committed to showcasing quality work across a wide-ranging set of bioethical issues, in an extensive set of countries, and using a diverse range of research methods. This is well-demonstrated in our Original Articles and Analysis sections. As to the former, Deshpande et al. (2020) offer the first of our non-COVID-19 contributions with their enlightening account of the challenges of conducting research on mental health in India; more generally, Das et al. (2020) mount a compelling case for the reuse of disposable medical supplies - a matter now made all the more pertinent during the COVID-19 crisis - and they provide a key set of ethical considerations that ought to be operative to support this practice. From the general to the specific, Muyskens (2020) invites us to consider whether and how Confucian ethics can address elder care challenges in Singapore, reminding us that the precepts of this philosophy are far more complex in their application to the modern world than one might imagine at first blush.

Our next two papers offer insights based on empirical research. Alone and together, they are excellent illustrations of this kind of quality research being conducted in Asia, and we welcome more contributions of this nature in the future. For the present issue, Hibino (2020) provides a fascinating account of the history and contemporary practice of noncommercial surrogacy in Thailand; in doing so, we are made witness to the infectious nature of human practices between and across cultures, as well as to the common ethical concerns that endure. This is reflected both in the cross-border challenges that Thailand has encountered, and in its attempts to provide robust ethical regulation of surrogacy as an international phenomenon. Our second qualitative contribution comes from Sasi et al. (2020) in the context of post mortem body donation in India. Set against prevailing cultural beliefs and practices that might sometimes militate against such donation, the authors deftly present cross-cutting perspectives and attitudes from donors, their families and religious scholars on these matters. The picture that emerges, as is so often the case with this kind of research, confounds many of our pre-existing expectations.

Our final Original Article in this issue comes from Bergstresser et al. (2020) who deliver something of a tour de force on medical ethics education by presenting a comparative analysis from a range of countries that is focussed on the normative importance of ensuring that the 'local' experience is properly integrated into training of future health care professionals. The June 2020 issue is completed with our "Analysis" 
section that contains the insights from our colleague, Saumil Dholakia (2020), about the on-going challenges of delivering universal healthcare in India. Recent legislative measures are subjected to analysis using the stakeholder method to assess them for their ethical robustness and sufficiency. There are important lessons here for all countries struggling with this elusive ideal of universal, fair and effective healthcare for all.

This issue stands as testament to the sustainability of bioethical contributions, both those that are a rapid response to a crisis such as COVID-19 and those that demonstrate the continued struggle we face collectively to bring about changes for the better in human health and well-being.

\section{References}

Bergstresser, Sara M., Kulsoom Ghias, Stuart Lane, Wee-Ming Lau, Isabel S.S. Hwang, Olivia M.Y. Ngan, Robert L. Klitzman, and Ho Keung Ng. 2020. What does it mean for a case to be "local"? The importance of local relevance and resonance for bioethics education in the Asia Pacific region. Asian Bioethics Review 12 (2). https://doi.org/10.1007/s41649-020-00120-8.

Cave, Emma. 2020. COVID-19 super-spreaders: Definitional quandaries and implications. Asian Bioethics Review 12 (2). https://doi.org/10.1007/s41649-020-00118-2.

Das, Anjan Kumar, Taketoshi Okita, Aya Enzo, and Atsushi Asai. 2020. The ethics of the reuse of disposable medical supplies. Asian Bioethics Review 12 (2). https://doi.org/10.1007/s41649-020-00114-6.

Deshpande, Smita N., Vishwajit L. Nimgaonkar, Triptish Bhatia, Nagendra Narayan Mishra, Rajesh Nagpal, and Lisa S. Parker. 2020. Ethical practices and legal challenges in mental health research in India. Asian Bioethics Review 12 (2). https://doi.org/10.1007/s41649-020-00116-4.

Dholakia, Saumil. 2020. An ethical analysis of the Ayushman Bharat Pradhan Mantri Jan Arogya Yojana (PM-JAY) using the stake-holder approach to universal health care in India. Asian Bioethics Review 12 (2). https://doi.org/10.1007/s41649-020-00121-7.

Gopichandran, Vijayaprasad, Sudharshini Subramaniam, and Maria Jusler Kalsingh. 2020. COVID-19 pandemic: A litmus test of trust in the health system. Asian Bioethics Review 12 (2). https://doi. org/10.1007/s41649-020-00122-6.

Hibino, Yuri. 2020. Non-commercial surrogacy in Thailand: Ethical, legal, social implications in local and global context. Asian Bioethics Review 12 (2). https://doi.org/10.1007/s41649-020-00126-2.

Krishna, Lalit Kumar Radha, Han Yee Neo, Elisha Wan Ying Chia, Kuang Teck Tay, Noreen Chan, Patricia Soek Hui Neo, Cynthia Ruth Goh-Fung, Tan Ying Peh, Min Chiam, and James Alvin Yiew Hock Low. 2020. The role of palliative medicine in ICU bed allocation in COVID-19: A joint position statement of the Singapore hospice council and the chapter of palliative medicine physicians. Asian Bioethics Review 12 (2). https://doi.org/10.1007/s41649-020-00128-0.

Lim, Hannah YeeFen. 2020. Ethics education for successful infectious disease control of COVID-19. Asian Bioethics Review 12 (2). https://doi.org/10.1007/s41649-020-00124-4.

Muyskens, Kathryn. 2020. Will Confucian values help or hinder the crisis of elder care in modern Singapore? Asian Bioethics Review 12 (2). https://doi.org/10.1007/s41649-020-00123-5.

Sasi, Aiswarya, Radhika Hegde, Stephen Dayal, and Manjulika Vaz. 2020. Life after death - The dead shall teach the living' - A qualitative study on the motivations and expectations of body donors, their families and religious scholars in the south Indian city of Bangalore. Asian Bioethics Review 12 (2). https://doi. org/10.1007/s41649-020-00117-3.

Xafis, Vicki. 2020. "What's inconvenient for you, is life-saving for me": How health inequities are playing out during the COVID-19 pandemic. Asian Bioethics Review 12 (2). https://doi.org/10.1007/s41649-02000119-1.

Xafis, Vicki, G. Owen Schaefer, Markus Labude, Yujia Zhu, and Li Yang Hsu. 2020. The perfect moral storm: Diverse ethical considerations in the COVID-19 pandemic. Asian Bioethics Review 12 (2). https://doi.org/10.1007/s41649-020-00125-3.

Publisher's Note Springer Nature remains neutral with regard to jurisdictional claims in published maps and institutional affiliations. 